\title{
MEASUREMENT OF LOCAL TEXTURES WITH TRANSMISSION AND SCANNING ELECTRON MICROSCOPES $\dagger$
}

\author{
R. A. SCHWARZER \\ Institut für Metallkunde und Metallphysik der TUC, Großer Bruch 23, \\ D-3392 Clausthal-Z., Germany
}

(Received February 5 1990)

\begin{abstract}
Transmission and scanning electron microscopy methods are discussed for the determination of grain orientations. For the study of local textures with a TEM electron-transparent thin samples are required. The standard techniques of orientation determination grain by grain are the interpretation of selected area electron spot and microbeam Kikuchi diffraction patterns. Specimen areas smaller than $500 \mathrm{~nm}$ or $50 \mathrm{~nm}$ in diameter can be selected. More recently selected area pole-figures can be measured directly with a TEM technique similar to the conventional transmission X-ray method.

The orientation of grains in a bulk sample can be obtained with a scanning electron microscope from reflection Kikuchi (i.e. electron backscattering) and channeling patterns. Local resolution is approximately $1 \mu \mathrm{m}$ or $5 \mu \mathrm{m}$, respectively.

Since the interpretation of electron diffraction patterns is tedious, techniques have been developed to perform measurements on-line by interfacing the electron microscope to a computer. An outstanding advantage of texture measurements by electron diffraction is the high local resolution and the ability of imaging the microstructure of the sampled region. Experimental results of individual grain-orientation measurements may be represented statistically by inverse pole-figures, orientation distribution functions and misorientation distribution functions.
\end{abstract}

KEY WORDS Electron diffraction, Kikuchi patterns, pole figure measurements, orientation distribution function, misorientation distribution, dual phase steel, titanium, shear bands

\section{INTRODUCTION}

Preferred crystal orientations (crystallographic "texture") are usually determined using X-ray or neutron diffraction methods. Hereby integration is performed over a large sample volume and hence over a large number of grains. These procedures are adequate for most applications, since texture determination is intended to get statistical information on the macroscopic behaviour or the previous history of the bulk material (Bunge, 1969). There are, however, some applications where the knowledge of local texture of areas smaller than $100 \mu \mathrm{m}$ diameter or even the orientations of individual grains are required, e.g. for a detailed understanding of deformation, recrystallization and grain growth.

$\dagger$ This paper was presented at the COMETT Symposium "Anisotropic and Microheterogeneous Materials", Metz, France, December 1989. 


\section{THE EVALUATION OF INDIVIDUAL GRAIN ORIENTATIONS}

\subsection{Selected-Area Diffraction in Transmission Electron Microscopy}

In selected-area diffraction mode (SAD) of the transmission electron microscope (TEM) the specimen is illuminated with a parallel beam of electrons, and an area of interest is selected from the enlarged image, with the help of a diaphragm in the intermediate image plane. The diffraction pattern from this area is displayed by focussing the back focal objective plane onto the fluorescence screen. Lateral resolution in SAD cannot be improved to any extent by simply stopping down the size of the selected area. As a consequence of the spherical and chromatic aberrations of the objective lens and unavoidable focussing errors, the dark-field images, which are formed on the selector diaphragm by the diffracted beams, are slightly displaced with respect to each other and to the bright-field image. The terms "bright-field" and "dark-field" refer to images to which the forwardscattered ("primary") beam does or does not contribute, respectively. In modern transmission electron microscopes the smallest selectable area in SAD is in the range of $200 \mathrm{~nm}$ to $500 \mathrm{~nm}$, as compared to $1 \mu \mathrm{m}$ to $1.5 \mu \mathrm{m}$ in older ones.

If the selected area is a single crystal, the diffraction pattern consists of bright regular spots ("spot pattern"). Since the sample must be rather thin to be transparent for electrons, the precision of measurement is reduced which results in an incorrect description of crystal orientation. Thus low-index orientations are emphasized so that real orientation distributions and particularly orientation relationships are obscured. In principle, angular accuracy of orientation may be improved from several degrees to about $0.5^{\circ}$, if both position and intensity of the diffraction spots are taken into account ("method of intensity center"; (Laird $e t$ al., 1966)).

\subsection{Microbeam Diffraction in the Transmission Electron Microscope}

With electron diffraction, unlike X-ray or neutron pole-figure measurements, it is possible to obtain information on a very localized region simply because the lens systems of the microscope allow the electron beam to be focussed to a small spot which defines the sampled area on the specimen. In modern TEM, particularly those equipped with a condenser-objective lens (e.g. PHILIPS EM 420/430 and CM series; JEOL FX series), the illuminated field can be made smaller at least by one order of magnitude than the smallest selectable region in SAD, and in addition the beam deflection coils can be used to move the spot onto the selected sample region (microbeam electron diffraction (MBD); "Feinstrahlbeugung" (Riecke, 1962)). With an intense focussed probe, however, a large angle of convergence of the beam cannot be avoided. Hereby disk-shaped rather than dotted diffraction spots, as with parallel illumination in SAD, are formed. This effect is expected to lead to a further loss of accuracy in evaluating crystal orientation. Fortunately another type of electron diffraction pattern, named after S. Kikuchi, is more likely to be generated even with thin samples as the beam is made more convergent.

Kikuchi patterns are analogous to Kossel patterns in X-ray diffraction. For a detailed understanding of the formation of Kikuchi patterns the kinematic theory of electron diffraction (Whelan, 1970; Reimer 1984) is required, while the geometry of Kikuchi lines can be well interpreted by a strongly simplified model (Kikuchi, 1928). The diffracting plane lies, as a straight line, almost midway 


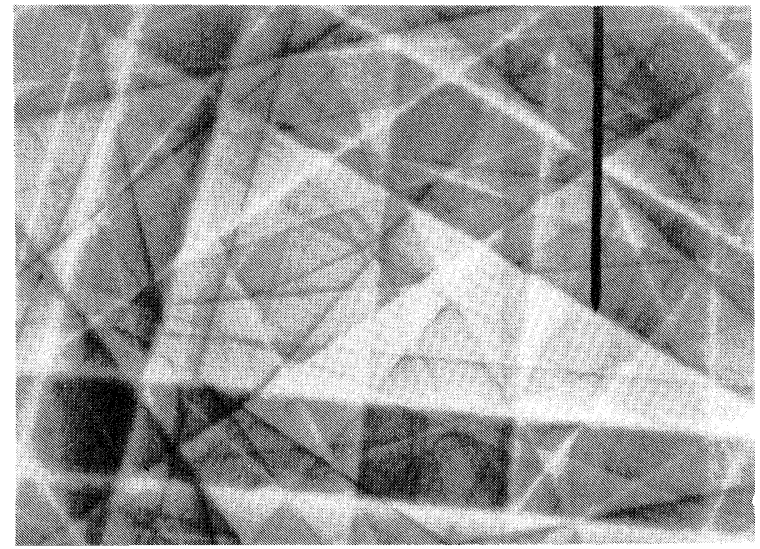

Figure 1 Kikuchi pattern from ferrite at $150 \mathrm{kV}$.

between the Kikuchi line pair which borders the Kikuchi band of angular width, $2 \theta$ (Figure 1). The angle of convergence of the primary beam is of little consequence here. So the often used term "convergent beam electron diffraction" (CBED) is not adequate here and should be reserved for applications when a large angle of convergence is essential (e.g. Kossel-Möllenstedt or HOLZ diffraction patterns (Reimer, 1984)).

Two conditions must be fulfilled for clear Kikuchi lines to be formed: First, there must be a sufficient number of electrons filling a large angular range along the primary beam direction in order to have a chance to be Bragg diffracted from obliquely oriented lattice planes. Secondly, there must not be significant differences in crystal orientation over the whole diffracting sample volume, otherwise the particular Kikuchi patterns from local orientations would merge and fade away on the background. The first condition is met either by extensive scattering of the primary beam when impinging on a thick specimen or, with a thin sample, by a convergent illumination (CBED). The second condition may be fulfilled with SAD only from large, perfect crystals or, with MBD, with a focussed electron beam of small spot size. When studying samples from heavily deformed materials, it is often essential to decrease the beam spot to less than $100 \mathrm{~nm}$ in diameter in order to select a column of the crystal that is not distorted, and free from dislocations. It is obvious that, by scattering the incoming electron beam, the foil thickness imposes a lower limit on the useful beam size. For the study of very thin foils it is advisable to lower the beam voltage and to increase the beam convergence as far as possible, since both means favour the formation of Kikuchi patterns. Accuracy of crystal orientation is limited by the sharpness of Kikuchi lines. If contiguous grains are studied, orientation differences less than $0.1^{\circ}$ can be detected. When evaluating the crystal orientation with respect to the workpiece, a larger error than this may be introduced by inaccurate marking of the reference directions when taking the sample from a bulk material, by deviations of the alignment of the sample holder or by local bending of the sample foil in the TEM. Using a scanning transmission electron microscope (STEM) channeling patterns can be obtained in transmission mode by an angular scan of the beam over the specimen (Figure 2). Approximately the same lateral and angular resolutions are reached as with microbeam transmission Kikuchi 


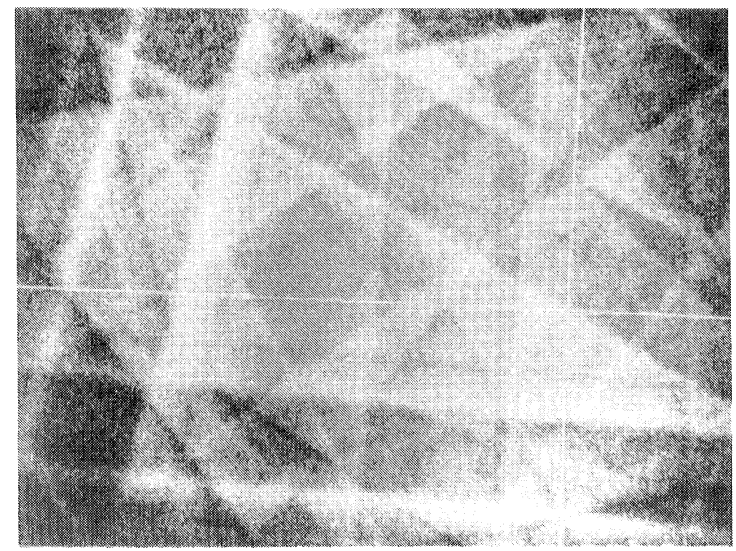

Figure 2 Channeling pattern from ferrite at $150 \mathrm{kV}$.

patterns. Since there is no obvious advantage over the transmission Kikuchi technique with stationary beam, no details are given here.

\subsection{The Determination of Grain Orientations with the Scanning Electron Microscope}

With a scanning electron microscope (SEM), grain orientations can be determined from reflection Kikuchi and channeling patterns. The mechanisms of the formation of these patterns differ somewhat from the generation of transmission Kikuchi patterns. The geometrical appearance (e.g. angular width of a band equals twice the Bragg angle) and the crystallographic information are so similar that virtually the same evaluation procedures can be applied. As with transmission Kikuchi patterns, clear channeling patterns are only formed if the diffracting volume is a perfect crystal. The blurr of the patterns is a measure of the internal stress (Davidson, 1984). Since the patterns are collected from the top layer of the sample down to less than about $2 \mu \mathrm{m}$ beneath the surface, special care has to be taken in preparing a clean surface free from foreign deformations. A deep etch or electrolytic polish of soft materials is advisable. A bibliography on the observation of crystalline materials by use of diffraction effects in the SEM is given elsewhere (Joy and Newbury, 1977). Crystal orientations of neighbouring grains can be obtained with an accuracy of about $0.5^{\circ}$, a value which is inferior to the accuracy of transmission Kikuchi patterns, due to the lower sharpness of the lines. Crystal orientations with respect to a frame fixed to the workpiece can be determined to about $1^{\circ}$ to $2^{\circ}$, since the positions of the samples on the stage and the primary electron beam may vary in this order of magnitude.

2.3.1. A reflection Kikuchi pattern is generated by focussing the stationary primary beam on a grain of interest in the surface of a bulk sample. For the acquisition in the SEM (Venables and Harland, 1973; Reimer and Grün, 1986) either a photographic plate or a fluorescent transmission screen is placed parallel to the incident beam, right in front of the tilted sample. In the latter arrangement the pattern may be recorded by a TV camera or by photography through a 


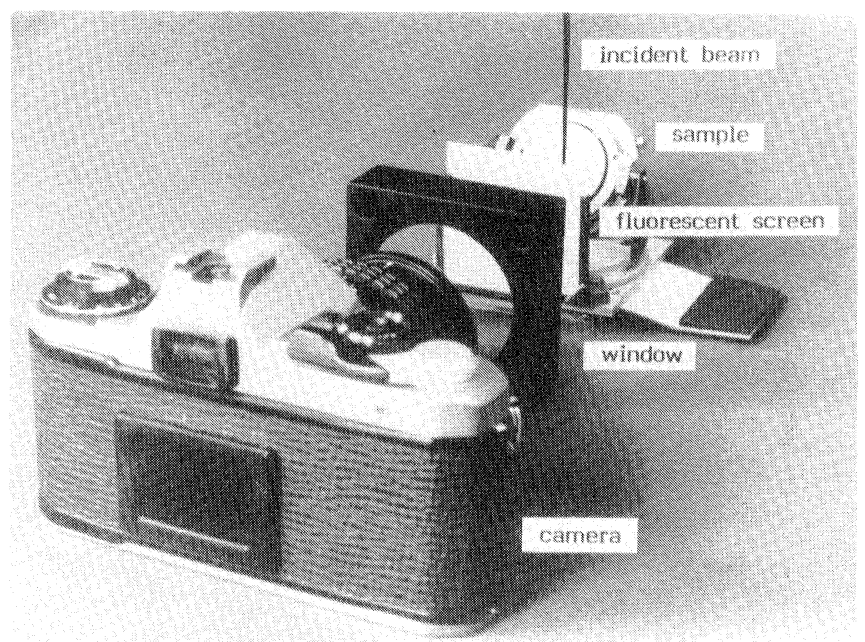

Figure 3 SEM set-up for recording reflection Kikuchi patterns.

window from outside (Figure 3). The stationary primary electron beam impinges on the sample at an angle of typically $30^{\circ}$ from grazing incidence. Reflection Kikuchi patterns when recorded on an SEM are also called electron backscattering patterns (EBS) (Venables and Harland, 1973) or backscatter Kikuchi patterns (Dingley, 1984).

The smallest detectable grain size corresponds to the slice of interaction volume cut away by the sample surface. Its shape is an elongated ellipse, due to the steep tilt of the specimen. Hence the diffracting volume is similar in diameter to lateral resolution in SEM micrography using backscattered electrons for imaging. With broad primary beams local resolution is approximately given by the spot size, whereas with fine beams the width of the probed slice lies between $0.2 \mu \mathrm{m}$ and $1.5 \mu \mathrm{m}$, depending on the accelerating voltage and the average sample density. Patterns from grains $0.2 \mu \mathrm{m}$ wide have been reported (Dingley, 1984). Advantages of reflection Kikuchi over channeling patterns are the very large range of recorded angles more than $\pm 20^{\circ}$ (which facilitates the consistent evaluation of grain orientation by merely considering zone-axis positions), undistorted patterns without image rotation due to a lensless geometric projection and a high probe brightness due to the large aperture of the incident beam. The focus settings and aperture stops of the microscope need not be adjusted when switching from imaging to diffraction mode. The beam spot has only to remain fixed on the selected grain, as with spot analysis in electron microprobe analysis.

2.3.2. Selected area channeling patterns (Coates, 1967; Brooker, 1970) from bulk samples are produced by the variation of the backscattered intensity with the angle between the incident beam and the crystal lattice. In the rocking beam method with pre-lens deflection, the scanning facility is used in combination with the probe-forming lens to scan the angle of beam incidence, with the pivot point resting stationary on the sample surface. For larger rocking angles the beam passes through the lens far from the optical axis. Mainly because of the spherical 
aberration and defocussing errors, the pivot point is widened to a disk. As a consequence of the long focal lengths and large spherical aberration coefficients of the probe-forming lenses, and the need for rocking angles in excess of $\pm 5^{\circ}$, the illuminated sample area is scarcely smaller than $10 \mu \mathrm{m}$ in commercial scanning microscopes. This is not sufficient for the study of cold worked or deformed materials. Spot sizes in the range of $1 \mu \mathrm{m}$ diameter (this corresponds to the minimum region selected in SAD, see 2.1) are possible by a modification (Joy and Newbury, 1972): The beam is scanned on a helix, and the probe-forming lens is refocussed according to the inclination between the beam and the optical axis such that the pivot point remains stationary. With the rocking-specimen set-up the sample is tilted mechanically under a stationary beam, using a eucentric goniometer stage. A large angular range of about $20^{\circ}$ with grains of $10 \mu \mathrm{m}$ diameter is reported (Brunner and Niedrig, 1979).

Channeling and reflection Kikuchi patterns are geometrically equivalent (Reimer, 1984). Due to the distortions of the scanning and recording systems, however, the accuracy of orientations obtained from channeling patterns falls behind reflection Kikuchi patterns. Channeling patterns can be obtained on several standard-version SEM. For high signal-to-noise ratios a backscatter detector (silicon diode or Robinson type system) is recommended. Reflection Kikuchi patterns require a special attachment to the SEM with the most expensive part being a low light level TV imaging system, to handle the low intensity and contrast of the patterns. An advantage over digital image store is to view the patterns with an integrating, Peltier cooled CCD camera (Schwarzer, 1989). The direct integration of faint, still patterns with the CCD sensor chip corresponds to long-term exposure on film in conventional microscopy. Since the patterns are collected from the top layer of the sample down to less than about $1 \mu \mathrm{m}$ beneath the surface, special care has to be taken in the preparation of a clean surface free from foreign deformations.

\section{ON-LINE MEASUREMENT AND INTERPRETATION OF ELECTRON DIFFRACTION PATTERNS}

The pairs of Kikuchi or channeling lines are fixed to their crystal lattice planes such that the whole system of lines will exactly follow any rotation or tilt movement of the crystal. Precise measurement of crystal orientations relies on this feature and on the sharpness of lines. In contrast, electron diffraction spots are fixed to the primary beam direction through Bragg's law. They rather change their intensities than their positions in the diffraction pattern when the crystal is tilted over a large range. The precise grain orientation can be obtained from spot patterns only by considering the positions and intensities of several spots, whereas from Kikuchi or channeling patterns simply the positions of at least three bands must be known. Usually lattice planes from different crystallographic zones contribute to a Kikuchi or channeling pattern. Hence they are, unlike spot patterns, not symmetric with respect to the primary beam spot unless the primary beam is incident along a symmetry zone axis, and $[u v w]$ and $[\bar{u} \bar{v} \bar{w}]$ directions can be distinguished from each other. The orientation of a crystal with respect to a reference frame (e.g. an orthogonal frame with the $x$ - and $y$-axes normal to the beam direction) can be unambiguously determined with high precision by evaluating a single Kikuchi pattern. On the other hand two complementing spot 
patterns are required in general, which must be taken at known specimen tilts. A further advantage of transmission Kikuchi over spot patterns is the wide range of useful sample thickness. Even with thick foils, close to the limit of transparency, Kikuchi patterns show sufficient contrast.

The wide application of Kikuchi and channeling patterns, particularly in the case of non-cubic crystal symmetry, is impeded by the tedious procedure of indexing. Only when the primary beam is incident close to a low-index direction do the patterns become so simple that they may be identified by visual inspection. Several procedures have been reported for the evaluation of Kikuchi (Newbury and Joy 1971; Heilmann et al., 1982; Hartig, 1984) and channeling (Ishida et al., 1981) micrographs. The measurements of diffraction coordinates have been facilitated by using a digitizing tablet, a projection device or a TV camera with image analyzer. The orientation determinations have been carried out with a separate computer off-line from the microscope.

A significant advance is to perform data acquisition and evaluation of diffraction spot (Carr, 1982), transmission Kikuchi or channeling (Schwarzer and Weiland, 1984) and reflection Kikuchi (Dingley, 1984) patterns on-line with a microcomputer linked to the microscope. Diffraction data may be acquired on-line in the TEM under operator control simply by deflecting the pattern over the fluorescent screen until selected features (diffraction spots or Kikuchi lines) are coincident with a reference mark (Figure 4) (Weiland and Schwarzer, 1985). The voltage drop over the beam deflection coils is directly passed on to the computer, as a measure of the selected position coordinates in the pattern. With the SEM a similar procedure is possible by placing a cursor at selected positions

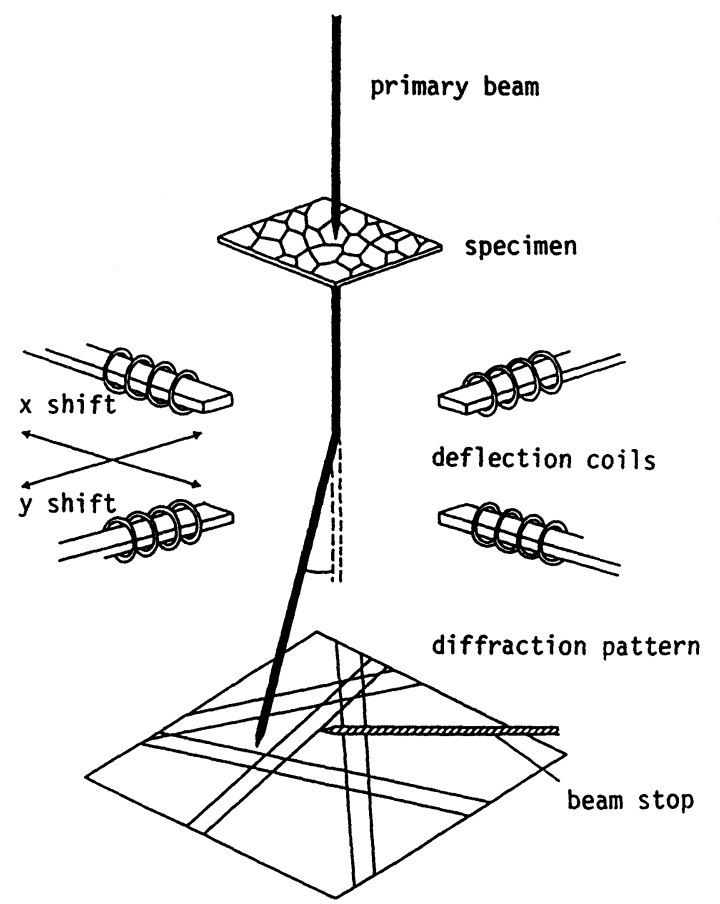

Figure 4 The on-line acquisition of Kikuchi patterns with a TEM. 


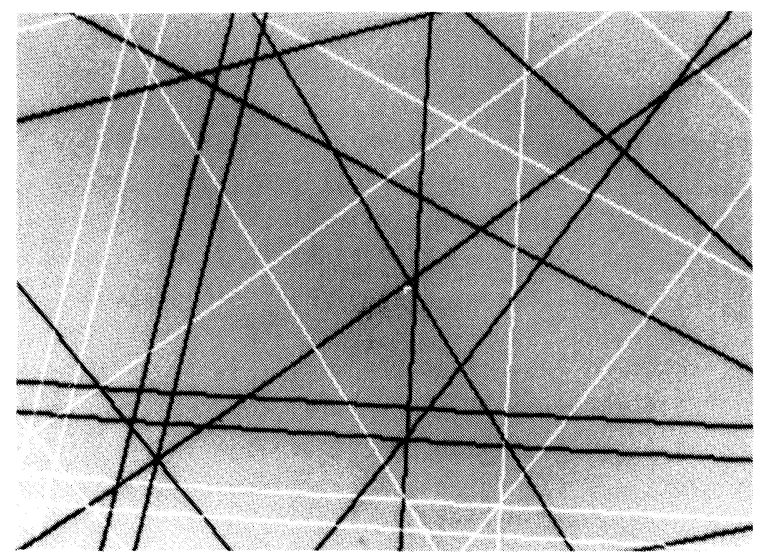

Figure 5 Recomputed Kikuchi map (to Figures 1 and 2). (Normalized beam direction $\langle 0.25,0.47,0.85\rangle$ ).

on a channeling pattern which is displayed on the SEM cathode-ray tube (Figure 2). After the interpretation of a pattern has been accomplished, the Kikuchi map (Figure 5) is computed from the particular grain orientation and displayed on the monitor screen for visual comparison, in order to exclude false solutions. Details of the program can be found elsewhere (Schwarzer, 1989). The operator using this semi-automated method has to select, with some skill, preferably strong and small bands, if possible not all from the same zone axis. The operator control is not really a disadvantage, since experience speeds up analysis and can only be replaced to some extent by more sophisticated hard- or software. Furthermore a fully automatic on-line technique is susceptible to erroneous data and requires more expensive equipment. Nevertheless some efforts have been made to automatically transmit the pattern from the microscope to the computer (Ishida $e t$ al., 1984).

Reflection Kikuchi patterns, when taken with a TV camera, are more diffuse and contain less details than transmission Kikuchi or channeling patterns such that in many cases only diffuse bands rather than individual lines are discernible. Then indexing can no longer rely on Bragg's equation. Furthermore accuracy of crystal orientation is markedly reduced to less than $1^{\circ}$ when clear Kikuchi lines are not available. The range of accepted diffraction angles, however, extends over more than $\pm 20^{\circ}$ on a usual set-up, and a large section of the standard orientation triangle is covered by a single reflection pattern. Hence indexing may be performed simply by comparing the positions of the principal zone axes in the actual pattern with those of a Kikuchi map from literature. A principal zone axis is indicated by the crossing-point of two or more bands. This simple indexing method by visual inspection is practicable for crystals with high symmetry (cubic, hexagonal).

In a similar way reflection Kikuchi patterns can be interpreted on-line with a computer using a semi-automatic procedure under operator control (Dingley, 1984): In a first step the experimental set-up is calibrated by collecting and digitizing a pattern from a silicon crystal mounted with the [001] direction normal to the specimen stage. The positions of the [001] and [112] zones are then 
marked on the TV screen with a cursor. From their positions a program calculates the actual diffraction length (specimen-to-screen distance) and stores this reference orientation. Then the orientation of a (cubic) sample crystallite may be determined by focussing the stationary beam on the grain of interest, collecting a reflection Kikuchi pattern and identifying two low-index zones (e.g. [112], [114] or [111]), by moving the cursor on the screen to their positions. An experienced operator may succeed in identifying principal zones even with lower symmetry crystals so that this semi-automated on-line technique may be extended to non-cubic materials. In order to exclude false orientations, e.g. due to mistaken input zones, or-if the selected zones are not lying on a common mirror plane-to discriminate $[u v w]$ from $[\bar{u} \bar{v} \bar{w}]$ directions, it seems even more necessary here than with transmission Kikuchi patterns, to recompute a Kikuchi map from the potential solution and to check it with the actual pattern.

In quantitative texture analysis the distribution of grain orientations is considered by number as well as by volume. The volume fraction and orientation of each grain is required. The surface and, making reasonable assumptions, the volume fraction of grains with a specific orientation can be determined simply by an additional stereological evaluation of the TEM or SEM image. To this end the grain-boundary coordinates may be acquired on-line, in a similar way as the data input is accomplished from diffraction patterns, by deflecting the TEM image with respect to a screen mark (Schwarzer and Weiland, 1987) or by following the grain boundary with the cursor on the SEM screen.

Table 1 recapitulates the features of on-line electron diffraction techniques for grain-specific orientation analysis and related applications. Typical values for local and angular resolution are given.

\section{REPRESENTATION OF INDIVIDUAL GRAIN ORIENTATIONS}

Experimental results of individual grain-orientation measurements are usually represented statistically by plotting the orientations for two reference directions in the workpiece point by point on inverse pole-figures (Figure 6). With a sufficiently great number of measured grains the points cluster together and form clouds around preferred orientations.

For comparison of individual grain orientation with X-ray pole-figure measurements it is convenient to compute the series expansion of the orientation distribution function (ODF) (Bunge, 1969; Wagner 1969). Figure 7 shows the ODF for 100 ferrite grains of a dual-phase steel sample obtained by individual grain orientation measurements (Schwarzer and Weiland, 1988). The volume fractions have been considered. It is worth noting that pole-figure measurements with X-rays cannot distinguish between the texture components of the ferrite and martensite phases.

For an illustration of the insufficient accuracy of SAD orientation measurements, the ferrite grain orientations have been converted to hypothetic SAD orientations by rounding off each exact primary beam direction into the nearest integer direction, making the assumption that spots up to $\{410\}$ were available for the interpretation of spot patterns. In spite of the fact that the SAD orientations differed less than $9^{\circ}$ from the original Kikuchi orientations, the ODF is markedly changed (Figure 8). The conclusion reached is that orientation measurements 
Table 1 Comparison of electron diffraction methods for texture analysis

\begin{tabular}{|c|c|c|c|c|}
\hline \multirow[t]{2}{*}{ Type } & \multirow[t]{2}{*}{ Equipment } & \multicolumn{2}{|l|}{ Resolution } & \multirow[t]{2}{*}{ Main applications } \\
\hline & & Local & Angular & \\
\hline Spot pattern & TEM (SAD) & $0.5-1.5 \mu \mathrm{m}$ & $5^{\circ}\left(1^{\circ}\right)$ & $\begin{array}{l}\text { thin film samples } \\
\text { dark field imaging } \\
\text { dislocations (weak beam) } \\
\text { Burgers vector analysis } \\
\text { estimate of orientation } \\
\text { precipitates }\end{array}$ \\
\hline \multirow[t]{2}{*}{$\begin{array}{l}\text { Transmission } \\
\text { Kikuchi } \\
\text { pattern }\end{array}$} & TEM (SAD) & $0.5-1.5 \mu \mathrm{m}$ & $0.1^{\circ}$ & $\begin{array}{l}\text { thick foil samples } \\
\text { coarse grains } \\
\text { orientation difference } \\
\text { dislocation density } \\
\text { internal stresses }\end{array}$ \\
\hline & $\begin{array}{l}\text { TEM (micro- } \\
\text { beam) } \\
\text { STEM }\end{array}$ & $10 \mathrm{~nm}$ & $0.2^{\circ}$ & $\begin{array}{l}\text { fine grains } \\
\text { deformed materials } \\
\text { grain specific texture } \\
\text { orientation difference } \\
\text { grain growth }\end{array}$ \\
\hline $\begin{array}{c}\text { Reflection } \\
\text { Kikuchi } \\
\text { pattern }\end{array}$ & $\begin{array}{l}\text { SEM + } \\
\text { dedicated } \\
\text { facility }\end{array}$ & $0.2-1.5 \mu \mathrm{m}$ & $1^{\circ}$ & $\begin{array}{l}\text { bulk, coarse grain sample } \\
\text { grain specific orientation } \\
\text { dynamic studies (hot-stage) } \\
\text { recrystallization } \\
\text { orientation difference }\end{array}$ \\
\hline $\begin{array}{l}\text { Channeling } \\
\text { pattern }\end{array}$ & $\begin{array}{l}\text { SEM (standard) } \\
\quad+\text { option }\end{array}$ & $\begin{array}{r}10 \mu \mathrm{m} \\
2 \mu \mathrm{m}\end{array}$ & $0.5^{\circ}$ & $\begin{array}{l}\text { semiconductor single crystals } \\
\text { bulk, coarse-grain samples } \\
\text { internal stress } \\
\text { orientation difference } \\
\text { grain-specific orientation }\end{array}$ \\
\hline
\end{tabular}
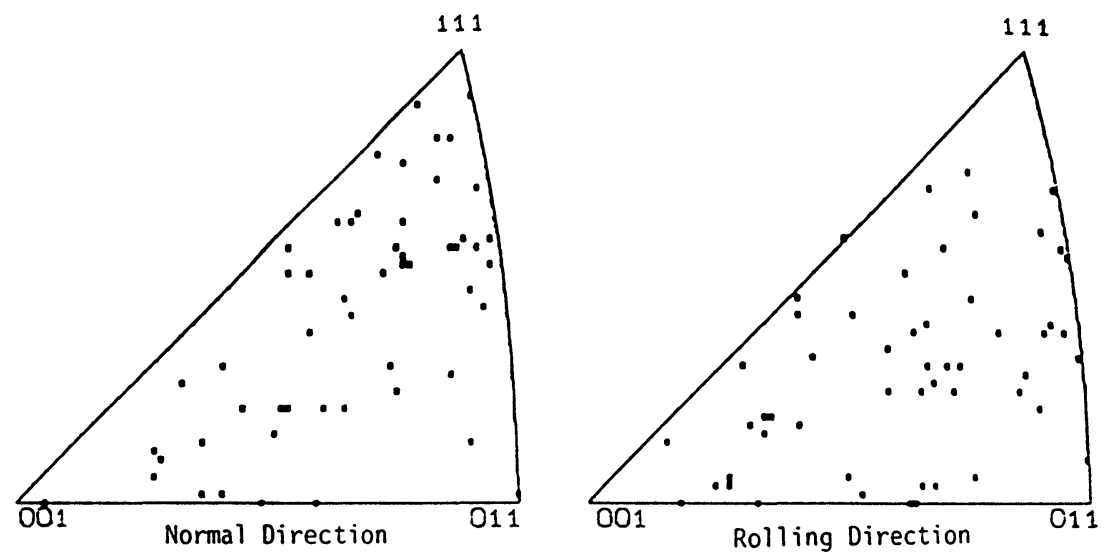

Figure 6 Inverse pole-figures from ferrite phase of a DP steel. 

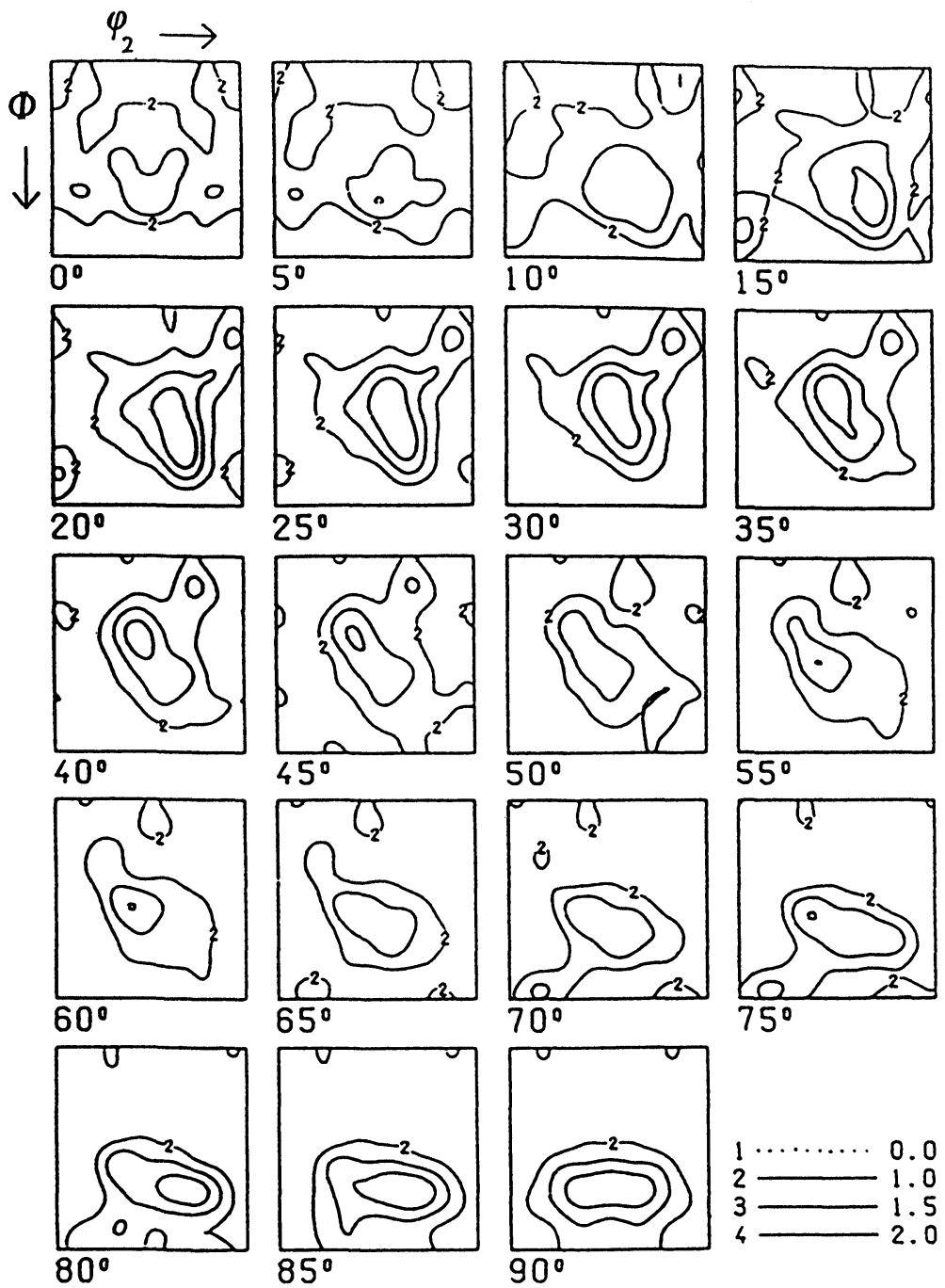

$75^{\circ}$

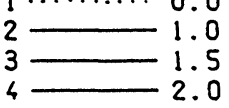

Figure 7 ODF of 100 ferrite grains in DP steel computed from Kikuchi patterns.

should be performed by the Kikuchi rather than the SAD method, particularly if orientation distributions and orientation correlations are studied.

Orientation differences, $\Delta g$ between individual grains, specified in Euler angles and superimposed by a Gaussian shaped peak, can be used for the series expansion of the misorientation distribution function (MODF), in a similar way as the series expansion of the ODF is performed (Plege, 1987; Bunge and Weiland, 1988). The distribution of special grain boundaries may so be revealed which significantly affect the material properties. 


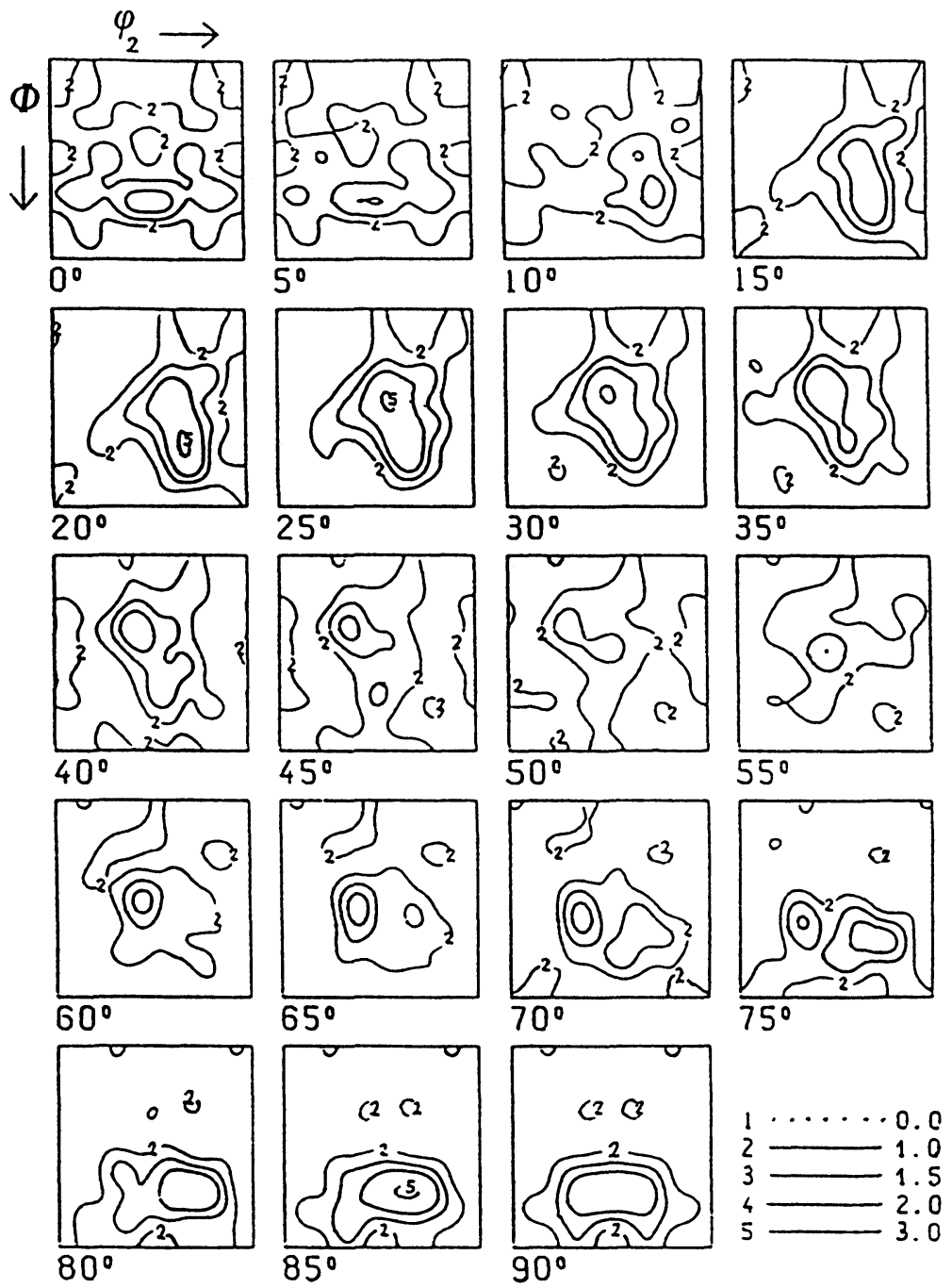

Figure 8 ODF calculated from simulated (SA] orientations of 100 ferrite grains in DP steel.

\section{ON-LINE MEASUREMENT OF POLE-FIGURES WITH THE TRANSMISSION ELECTRON MICROSCOPE}

Orientation measurements grain by grain yield information on the microtexture which is not obtained by any other technique. Considering the high expense, however, in sample preparation and equipment, the determination of individual orientations grain by grain using electron diffraction will be reserved for particular applications only. Extremely fine-grain or strongly deformed materials are still not accessible to single orientation measurements, since the grains may 
not be distinguished from each other, or the patterns are to diffuse. In addition the knowledge of pole-figures rather than grain specific texture is often sufficient. This led to the development of a technique for direct measurement of pole-figures with the TEM, independently in London (Humphreys, 1983) and Clausthal (Schwarzer, 1983, 1985).

The measurement of TEM pole-figures by electron diffraction is based on the same principles as the conventional film technique with X-ray diffraction. A main conceptual difference comes from the considerably shorter wavelength of energetic electrons (typically several pm) and hence the small Bragg angles of less than about $2^{\circ}$. If the sample is not a single crystal the diffraction pattern consists of arced reflections which fill more or less the Debye-Scherrer rings, according to the spread of grain orientations around the incident beam. So in general a single diffraction pattern may not reveal the true texture of the workpiece, since only the preferred orientations are represented whose lattice planes are close to the primary beam direction. A series of diffraction patterns must be considered while tilting the sample through large angles. Two automatic techniques have been developed for the on-line measurement of diffracted intensities (Schwarzer, 1985):

- Circular scan of the diffraction patterns over a Faraday cup by a postspecimen deflection of the beam

- Conical deflection of the primary beam in front of the specimen, and acquisition of intensity values from diffraction patterns or dark-field images using the Faraday cup or the fluorescent screen, respectively.

In both versions the specimen is tilted at small angular steps, $\Delta \beta$, along a preferred axis perpendicular to the primary beam direction (e.g. the rolling direction which is placed parallel with the goniometer axis) (Figure 9), while the electronic deflection may be done with the same angular increments or continuously. The angle of mechanical tilt, $\beta$, and the deflection angle, $\alpha$, define a

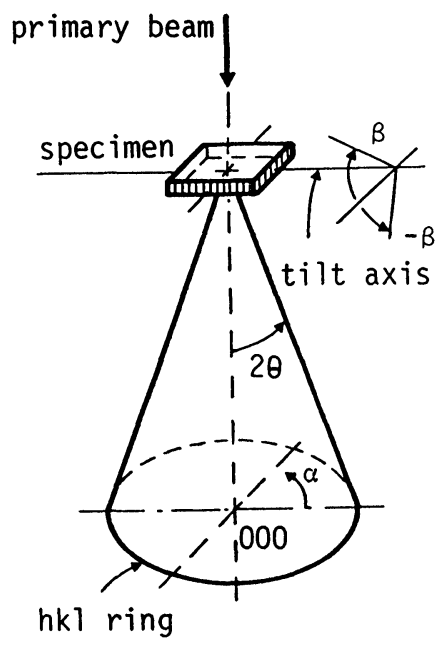

Figure 9 Schematics of set-up for TEM pole-figure measurement. 
point on the pole-figure for which the diffracted intensity $I(\alpha, \beta)$ is measured on the selected hkl ring. Since the sample soon becomes impervious with steep inclination, the angle of tilt ranges up to only $45^{\circ}$ or $60^{\circ}$. Hence TEM pole-figures are incomplete with a lens-shaped center not accessible to measurement. The blind center may be reduced to a barrel-shaped area by measuring one more pole-figure, with the axis of mechanical tilt rotated by $90^{\circ}$, and superposing both pole-figures correctly. Local texture can be measured from areas as small as the finest specimen regions selectable by SAD $(0.2 \mu \mathrm{m}$ to $1.5 \mu \mathrm{m}$ diam.), provided the number of sampled grains is statistically sufficient.

\section{QUANTIFICATION OF TEM POLE-FIGURES}

The diffracted intensities must be corrected in order to yield the distribution of crystallographic poles ("texture"), since they do not depend only on the volume fraction of crystallites with lattice planes of the appropriate orientations, but furthermore vary with crystal thickness, with the inclination of the sample, with slight deviations from the exact Bragg orientation, with multiple scattering and many-beam interactions. Two program options have been developed to interpret quantitatively the diffracted intensities in terms of pole-volume distribution (Schwarzer, 1989). With the "analytical correction," allowance is made for the

(a)
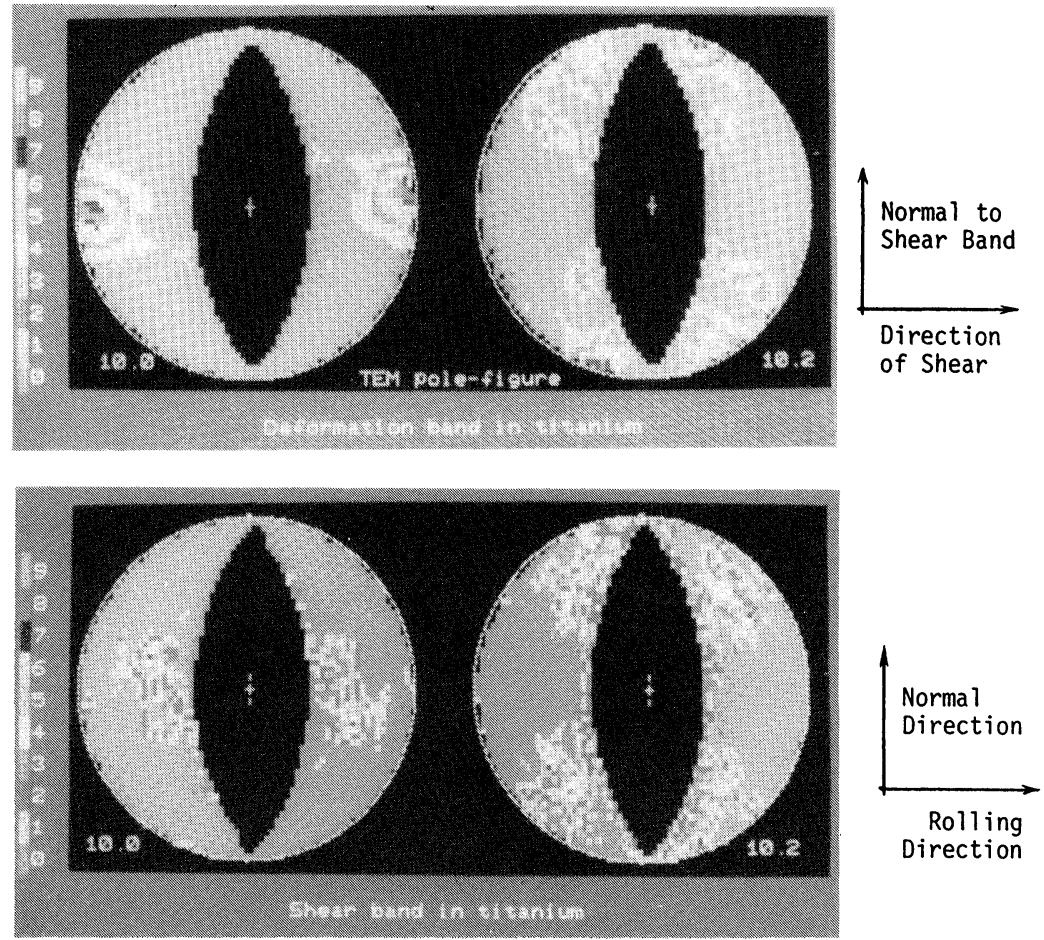

(b)

Figure 1010.0 and 10.2 TEM pole-figures from (a) deformation and (b) shear band in titanium after $80 \%$ deformation by cold rolling. 
increase of diffracting volume and of absorption when tilting the sample out of the normal incidence of the beam. The fitting parameters were determined by comparison with pole-figures from randomly oriented test samples of aluminium and copper. With the "experimental correction" the intensities of each Debye-Scherrer ring are normalized with respect to its values at the intersections of the tilt axis. These reference values belong to virtually the same point on the pole-figure. Hence their variations, when tilting the sample, reveal the effects which do not originate from crystallographic texture (Schwarzer, 1983).

\section{EXAMPLE OF APPLICATION OF TEM POLE-FIGURES}

TEM pole-figures were measured, at selected areas of some microns in diameter, from deformation and shear bands after $80 \%$ deformation of titanium by rolling (Schwarzer, 1988). Deformation bands show the usual crystallographic texture of titanium sheet known from X-ray measurements, whereas the texture of shear bands is distinctly different. The ideal orientation in deformation bands can be expressed by (00.1) [10.0] rotated around the rolling direction at about $40^{\circ}$ out of the transverse direction (Figure 10a). The ideal orientation for a shear band can be described by crystals with their [10.0] axes parallel with the transverse direction. They are rotated at about $30^{\circ}$ to $40^{\circ}$ out of the rolling direction (Figure $10 \mathrm{~b})$ such that the grains are preferably oriented with the $c$-axes parallel with the normal on the shear-plane.

\section{References}

Brooker, G. R. (1970). In: Amelinckx, S., Gevers, R., Remant, G., and Van Landuyt, J., (eds.), Modern Diffraction and Imaging Techniques in Materials Science, North-Holland Publ. Company, Amsterdam-London, pp. 613-653.

Brunner, M. and Niedrig, H. (1979). Beitr. elektr. Direktabb. Oberfl. BEDO 12/1, 333-338.

Bunge, H.-J. (1969). Mathematische Methoden der Texturanalyse, Akademie-Verlag Berlin.

Bunge, H. J. and Weiland, H. (1988). Textures and Microstructures 7, 231-263.

Carr, M. J. (1982). JEOL news 20E, 7-9.

Coates, D. G. (1967). Phil. Mag. 16, 1179-1784.

Davidson, D. L. (1984). Intern. Metals Review 29, 75-95.

Dingley, D. J. (1984). Scanning Electron Microscopy 1984/II, 569-575.

Hartig, Ch. (1984). Elektronenmikroskopische Untersuchung der Deformations-Texturen und des Beginns der Rekristallisation von plastisch verformten Ni-Einkristallen, Ph. D. Thesis, TU Berlin.

Heilmann, P., Clark, W. A. T. and Rigney, D. A. (1982). Ultramicroscopy 9, 365-372.

Humphreys, F. J. (1983). Textures and Microstructures 6, 45-62.

Ishida, Y., Mori, M., Arimoto, A. and Onoe, M. (1981). Proc. 6th Intern. Conf. on Textures of Materials, ICOTOM 6, 601-608.

Joy, D. C. and Newbury, D. E. (1977). SEM/1977 Vol. I: Proc. Workshop Analytical Electron Microscopy, ed. Johari, O., (IITRI Chicago), 445-454.

Joy, D. C. and Newbury, D. E. (1972). J. Material Science 7, 714-716.

Kikuchi, S. (1928). Jap. J. Physics 5, 83-96.

Laird, C., Eichen, E. and Bitter, W. R. (1966). J. Applied Phys. 37, 2225-2231.

Newbury, D. E. and Joy, D. C. (1971). Proc. 25th Anniversary Meeting of EMAG, Inst. Physics, 306-309.

Plege, B. (1987). In: Bunge, H. J., (ed.): Theoretical Methods of Texture Analysis, DGM Informations-Ges. Oberursel, pp. 393-403.

Reimer, L. (1984). Transmission Electron Microscopy, Springer-Verlag Berlin-Heidelberg-New York-Tokyo. 
Reimer, L. and Grün, D. (1986). BEDO 19, 105-110.

Riecke, W. D. (1962). Optik 19, 19-116.

Schwarzer, R. (1983). BEDO 16, 131-134.

Schwarzer, R. and Weiland, H. (1984). Proc. 7th Intern. Conf. on Textures of Materials, ICOTOM 7 , pp. 839-843.

Schwarzer, R. (1985) BEDO 18, 61-68.

Schwarzer, R. A. and Weiland, H. (1987). Proc. 8th Intern. Conf. on Textures of Materials, ICOTOM 8, pp. 203-208.

Schwarzer, R. A. and Weiland, H. (1988), Textures and Microstructures 8\&9, 551-577.

Schwarzer, R. A. (1988). Proc. EUREM 88, Inst. Phys. Conf. Ser. No. 93 Vol 2, pp. 23-24.

Schwarzer, R. (1989). Die Bestimmung der lokalen Textur mit dem Elektronenmikroskop, Habilitation Thesis, TU Clausthal

Schwarzer, R. (1989). BEDO 22, 279-282.

Venables, J. A. and Harland, C. J. (1973). Phil. Mag. 27, 1193-1200.

Wagner, F. (1986). In: Bunge, H.-J. (ed.), Experimental techniques of texture analysis, DGM Informations-Ges. Oberursel, pp. 115-123.

Whelan, M. J. (1970). In: Amelinckx, S., Gevers, R., Remant, G., and Van Landuyt, J., (eds.), Modern Diffraction and Imaging Techniques in Materials Science", North-Holland Publ. Company, Amsterdam-London, pp. 35-98.

Weiland, H. and Schwarzer, R. (1985). BEDO 18, 55-60. 